\title{
The scintillation of GEMS coated with wavelength shifters
}

\author{
F.A.F. Fraga ${ }^{a} *$, S.T.G. Fetal ${ }^{\mathrm{a}}$, M.M.F.R. Fraga ${ }^{\mathrm{a}}$, E.F.S. Balau ${ }^{\mathrm{a}}$, \\ L.M.S. Margato ${ }^{a}$, R. Ferreira Marques ${ }^{a}$, A.J.P.L Policarpo ${ }^{a}$, F. Sauli ${ }^{\text {b }}$ \\ ${ }^{\text {a }}$ LIP - Coimbra and Departamento de Física da Universidade de Coimbra, Coimbra 3004-516, Portugal \\ ${ }^{\mathrm{b}}$ CERN, Geneva, Switzerland
}

\begin{abstract}
The optical readout of GEMs, presented a few years ago, is currently being considered for several detector projects. Although using suitable gaseous mixtures the GEM avalanches emit a large number of photons in the visible and/or NIR regions, many gaseous mixtures of great interest for detectors scintillate mainly in the UV, and the readout of these detectors is usually inefficient or very expensive. In this work we report on the operation of GEMs coated with a wavelength converter. We show that using a GEM with an optimal geometry the wavelength conversion process can be quite efficient and does not degrade the intrinsic position resolution of the detector, an usual drawback of the utilisation of wavelength shifters.
\end{abstract}

(C) 2004 Elsevier B.V. All rights reserved.

PACS: 29.40.Cs; 29.40.Cx

Keywords: GEM detectors; Wavelength shifter; Scintillation

\section{Introduction}

In recent years our group has presented data on the luminiscence of GEMs and its applications [14]. It was shown that if suitable mixtures are chosen, this type of devices readout by CCDs can be used for X-ray, thermal neutron and even used to develop charged particle tracking chambers. These applications were developed using either visible-NIR or enhanced readouts with sensitivity down to $200 \mathrm{~nm}$. In the first case, a large number

\footnotetext{
*Corresponding author. Tel.: +351-39-833-465; fax: +35139-822-358.

E-mail address: francisco@lipc.fis.uc.pt (F.A.F. Fraga).
}

of photons was lost, reducing the signal-to-noise ratio of the system, and although the more expensive CCD systems were sensitive over a spectral zone of high luminosity, the larger emission was compromised by the lower efficiency of the UV optics. It should also be considered that, although modern back illuminated CCD systems have very high sensitivity in the UV, down to $200 \mathrm{~nm}$, and their cost is decreasing, the UV optical systems are very expensive and inefficient.

The use of light converters or wavelength shifters in scintillation detectors has been studied for a long time. These substances must have high conversion factors, fast decay time, optical transparency in thin layers, low saturated vapour 
pressure, good mechanical strength and chemical stability [5]. Usually deposited on the internal side of the output window of the scintillator their use was quite successful for energy measurements and triggering purposes and it is still used to ameliorate the efficiency of detectors [6], but due to the distance between the scintillation and absorption and emission process zone they diminish the intrinsic position resolution of the detector.

Considering that the GEM scintillation is isotropic and localized in the GEM holes, if we could deposit the wavelength shifter on the inside walls of the channel the position resolution degradation could be overcome, keeping a reasonable efficiency. Also, if using a readout sensitive in both the scintillation and the wavelength shifter spectral zones, the overall efficiency of the system could be enhanced in a considerable way.

In this paper we report the results obtained while depositing P-terphenyl on the outer surface of a GEM.

\section{Experimental system}

A cross-section of the chamber is shown in Fig. 1. No collecting electrode was used in these experiments, avalanche electrons were collected by the GEM outer electrode. The entrance window was made from $12 \mu \mathrm{m}$ aluminized mylar foil and a $10 \times 10 \mathrm{~cm}^{2}$ GEM foil was used for the measurements. The GEM and the drift grids were supported by fiber glass frames. The light window

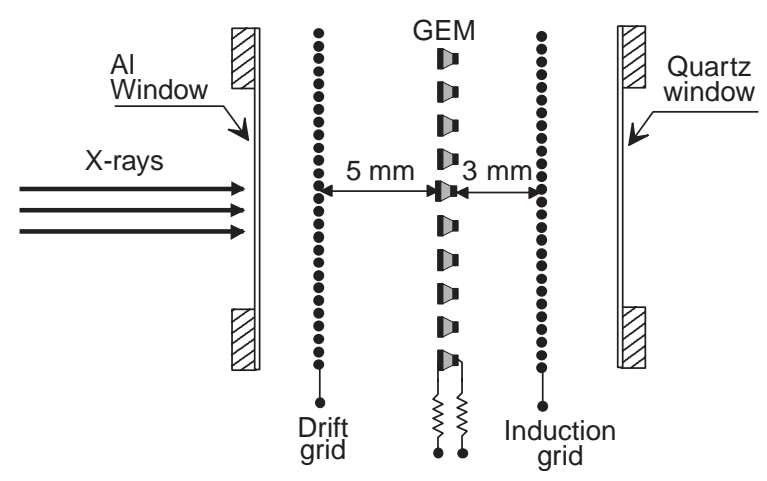

Fig. 1. Cross-section of the used detector. was a $6 \mathrm{~cm}$ diameter quartz window covering all the surface of the $3 \mathrm{~cm}$ diameter GEM. All these structures were clamped by two external aluminium frames.

The GEMs, supplied by the CERN GDD Group, were manufactured from a $50 \mu \mathrm{m}$ thickness copper coated kapton foil by a chemical etching process that produces holes with a double conical shape (standard shape) and conical shape (Fig. 2) [7]. The diameters of metal and kapton holes were 80 and $50 \mu \mathrm{m}$, respectively. The pitch was $140 \mu \mathrm{m}$ and the optical transparency $\approx 12 \%$. The GEM front side was grounded, the back one being operated at negative voltage.

A Hamamatsu UV CCD Hamamatsu C4880-21 was used to read the light emitted from the GEM. It uses a Peltier-cooled low noise CCD, and the spectral response goes from 200 to $1000 \mathrm{~nm}$. All the pictures presented in this work were taken with the CCD cooled to $-30^{\circ} \mathrm{C}$, without any fluid cooling.

A diagram of the complete system is shown in Fig. 3. The primary electrons produced by the Xray photons in the conversion region drift towards the GEM holes. The grid currents were monitored with the high-voltage power supply ammeters with

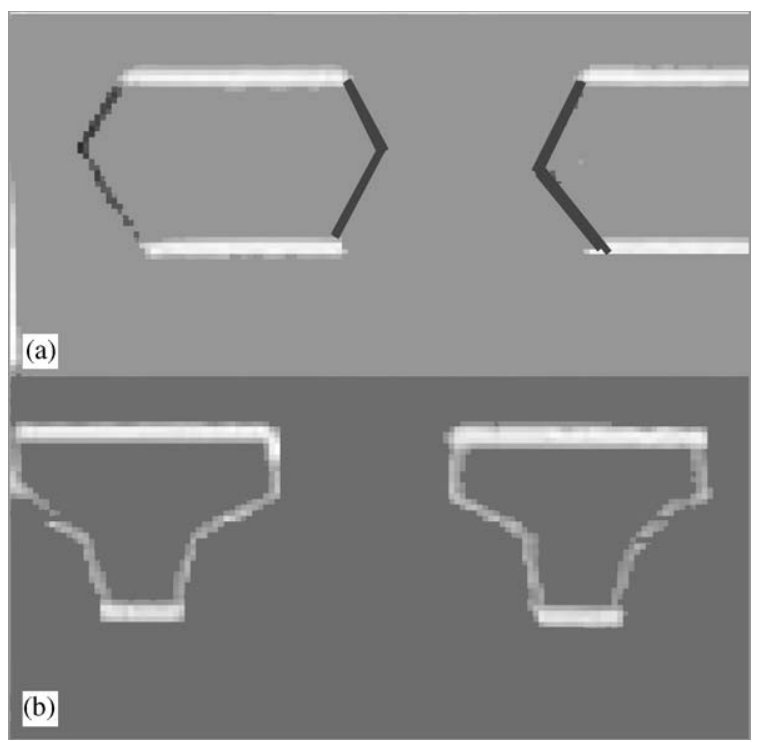

Fig. 2. Electron microscope cross-section of the double-conical or standard (a), conical (b) GEMs. 


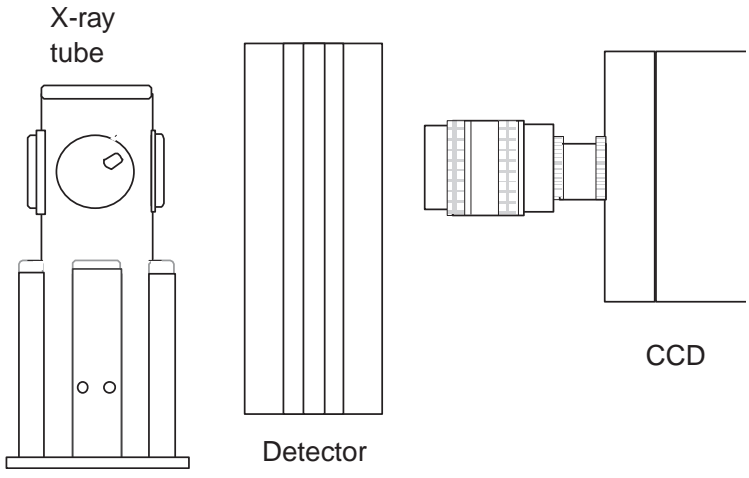

Fig. 3. Sketch of the experimental system used to make CCD images of the GEM scintillation.

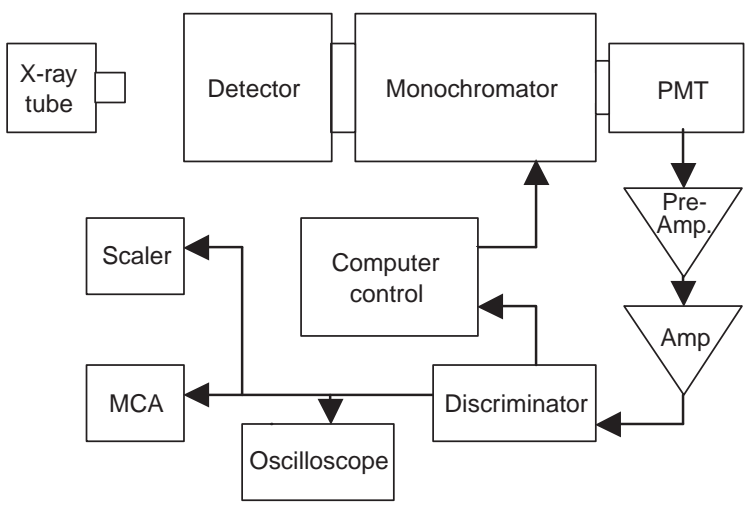

Fig. 4. Spectroscopic studies set-up.

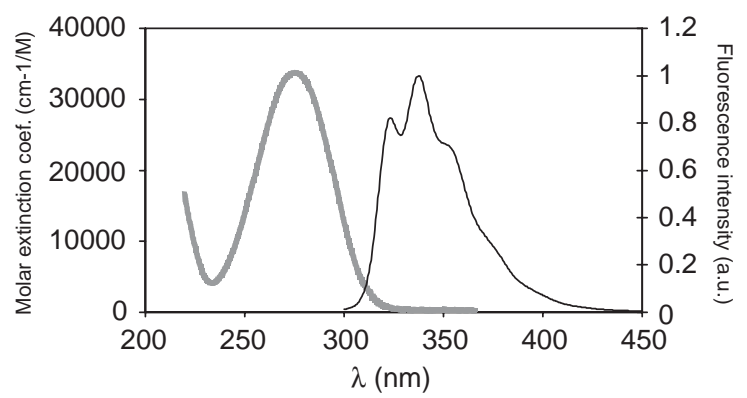

Fig. 5. P-terphenyl absorption and emission spectrum.

a resolution of $\mathrm{nA}$ and the outer GEM grid was connected to the ground through a Keithley picoammeter, allowing for precision measurement of the collected electrons current. All the electrons produced in the multiplication zone were collected

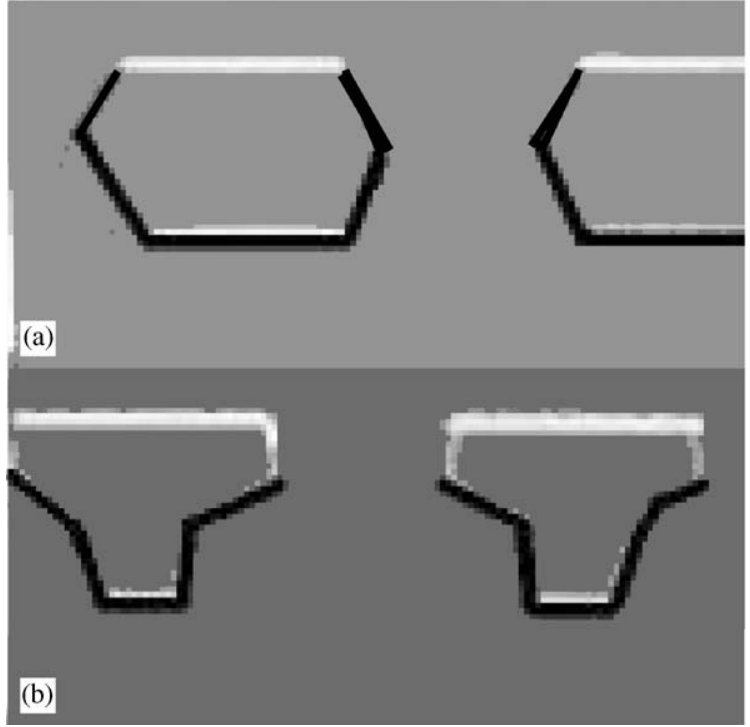

Fig. 6. Cross-section of the used GEMs showing the layer of Pterphenyl.

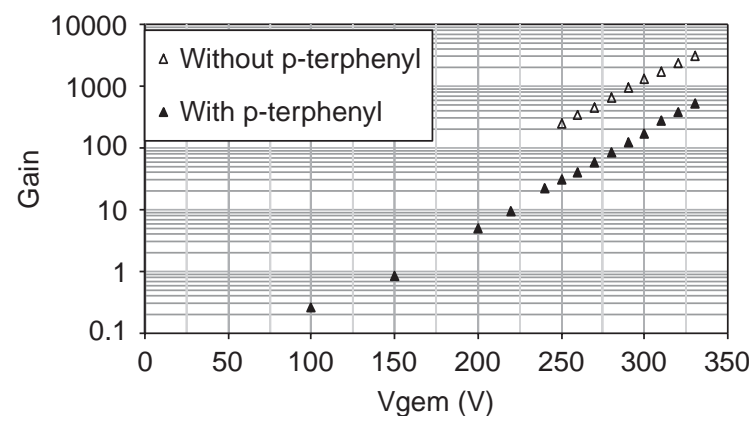

Fig. 7. Operation of GEMs coated with P-terphenyl in Ar$2.7 \%$ TEA.

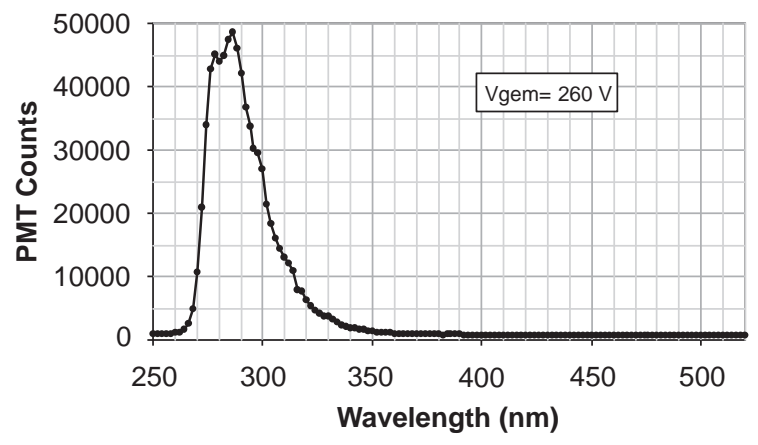

Fig. 8. Spectrum of Ar-2.7\% TEA in bare GEMs. 
at the outer electrode of the GEM and the ratio between primary current and this current was used to calculate the GEM gain.

In order to perform spectral studies, the light was analysed by an Applied Photophysics mono-

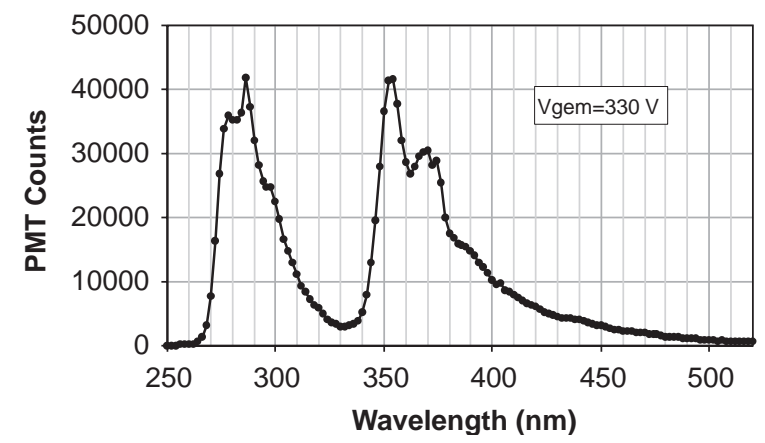

Fig. 9. Spectrum of Ar-2.7\% TEA in GEMs coated with Pterphenyl. chromator, mod. 7300, equipped with a $1200 \mathrm{~g} /$ $\mathrm{mm}$ grating blazed at $500 \mathrm{~nm}$ and detected by a RCA C31034A photomultiplier, cooled down to $-20^{\circ} \mathrm{C}$ and operated in the single photon counting mode (Fig. 4).

$\mathrm{X}$-ray photons from a ${ }^{55} \mathrm{Fe}$ source $(5.9 \mathrm{keV})$ and from an X-ray generator with a copper target are used as an excitation source. The tube is operated at $10 \mathrm{kV}$.

The detector was operated with an Ar- $2.7 \%$ TEA mixture, the emission spectrum is shown in Fig. 8.

The chosen wavelength shifter was P-terphenyl. Fig. 5 shows P-terphenyl absorption and emission, the absorption band reasonably overlaps the emission of the Ar-TEA mixture. An optimum thickness on $1 \mathrm{mg} / \mathrm{cm}^{2}$ was applied by evaporation to the outer side of the GEM facing the quartz output window.

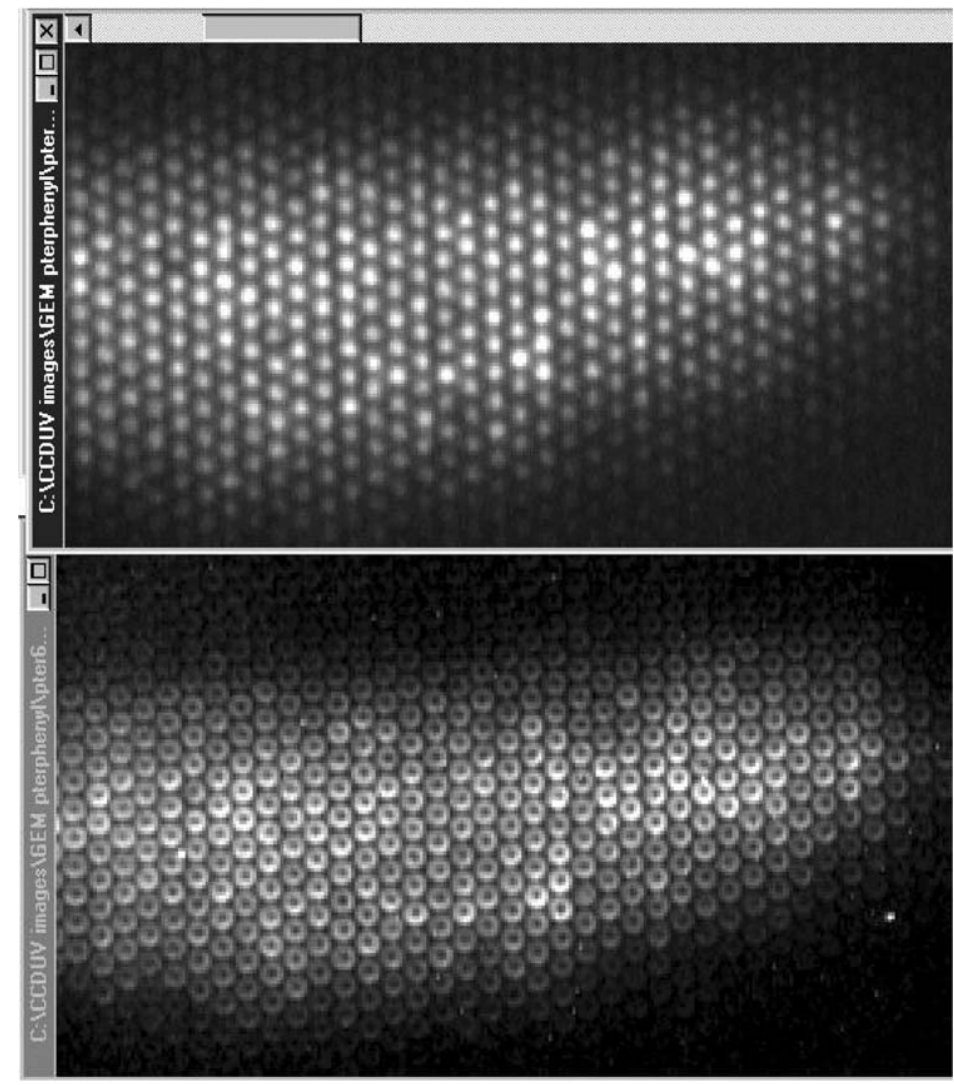

Fig. 10. CCD images of uniformly irradiated GEMs: (top) full spectrum and (bottom) bandpass filter. 


\section{Results and discussion}

The first tests were made with a standard biconical GEM and showed some emission in the $350 \mathrm{~nm}$ zone, but it was soon concluded that much better results could be anticipated using conical GEMs as shown in Fig 6. as the area covered with the P-therphenyl would be larger. All the results shown were obtained with the conical GEMs .

Fig. 7 shows data on the operation of the bare and coated GEM. As expected voltage had to be increased after the P-terphenyl was deposited, but safe usable gains of several hundred could be reached with the coated GEM. The well-know spectrum of the Ar-TEA mixture is shown in Fig. 8.

The untreated emission spectrum of $\mathrm{Ar}-2.7 \%$ TEA obtained with a GEM coated with P-terphenyl is shown in Fig. 9. The amplitude of both the ArTEA and P-terphenyl peaks is similar showing that the efficiency of the conversion process is high.

Fig. 10 shows two CCD images of the GEM scintillation taken with the full spectrum (top) and using a narrow bandpass $(\sim 10 \mathrm{~nm})$ sharp filter (bottom) centred at $400 \mathrm{~nm}$. The full spectrum image shows that the photon emission is localized in the channel zone and the ring structure obtained with the bandpass filter confirms that, as expected, the light from the P-terphenyl comes from the deposit along the conical holes area, and since the avalanche is located inside the channel, no photons are emitted by the GEM surface.

\section{Conclusions}

It was shown that GEMs can be operated with a wavelength converter and that by using optimal geometry the process becomes quite efficient. The data also suggests that the intrinsic position resolution of the GEM is unaltered by the shifting wavelength.

These results are of great interest as most noble gases and mixtures luminesce in the ultraviolet region and it is foreseen that this technique will originate the development of imaging chambers with high photon emission using much simpler and cheaper high-resolution readouts.

\section{References}

[1] F.A.F. Fraga, et al., Nucl. Instr. and Meth. A 471 (2001) 125.

[2] F.A.F. Fraga, L.M.S. Margato, S.T.G. Fetal, R. Ferreira Marques, A.J.P.L. Policarpo, IEEE Trans. Nucl. Sci. NS-49 (1) (2002) 281.

[3] F.A.F. Fraga, L.M.S. Margato, S.T.G. Fetal, Nucl. Instr. and Meth. A 513 (2003) 379.

[4] M.M.F.R. Fraga, R. Ferreira Marques, A.J.P.L. Policarpo, B. Guerard, A. Oed, G. Manzini, T. van Vuure, Nucl. Instr. and Meth. A 478 (2002) 357.

[5] S.A. Baldin, V.V. Matveev, Instr. Exp. Tech. (URSS) 4 (1963) 589.

[6] P. Bauleo, A. Goobar, J. Rodriguez Marino, Nucl. Instr. and Meth. A 443 (2000) 136.

[7] F. Sauli, Nucl. Instr. and Meth. A 386 (1997) 531. 